\title{
A spatial mixture approach to inferring sub-ROI spatio-temporal patterns from rapid event-related fMRI data
}

\author{
Yuan Shen ${ }^{1}$, Stephen Mayhew ${ }^{2}$, Zoe Kourtzi ${ }^{2,3}$, and Peter Tiňo ${ }^{1}$ \\ 1 School of Computer Science, University of Birmingham, United Kingdom \\ ${ }^{2}$ School of Psychology, University of Birmingham, United Kingdom \\ 3 Laboratory for Neuro- and Psychophysiology, K.U.Leuven, Belgium
}

\begin{abstract}
Previous works investigated a range of spatio-temporal models for fMRI data analysis to provide robust determination of functional region-of-interest (ROI). We present a novel spatio-temporal fMRI model that is suitable for identifying a number of distinct temporal patterns and their spatial support in the voxel space. Accordingly, fMRI signals on a single voxel are modeled as a probabilistic superposition of those temporal patterns. The spatially varying influence of individual patterns is defined in terms of a parameterised function. The temporal pattern is characterised by both the underlying hemodynamic response function (HRF) and a time series of the individual stimulus-response magnitudes, which makes the proposed model particularly suitable for modeling rapid event-related fMRI data. Moreover, a parametric approach is adopted to represent the HRFs. The resulting methodology is conceptually principled and computationally efficient. We first verify the proposed model in a controlled experimental setting using synthetic data. The model is further applied to analyzing real fMRI data, with focus on functional homogeneity within individual ROIs.
\end{abstract}

\section{Introduction}

The primary aim of fMRI data analysis is the detection of activated brain areas in response to given stimulus types, usually referred to as region of interest (ROI), through estimating the neural response level over the voxel space from fMRI data. Due to low sampling resolution and poor signal-to-noise ratio, a robust detection of neural activation requires spatial regularisation of the estimation. Moreover, the activation detection is intrinsically related to modeling and estimation of the underlying hemodynamic response function (HRF). Thus, a range of spatio-temporal fMRI models has been investigated over the past decade [1-5]. All these models are used to determine functional ROIs in whole brain fMRI analysis. It is usually accepted that a ROI is functionally homogeneous and the HRF remains invariant across all voxels within the region. If the primary goal of analysis is to extract sub-ROI information, the above assumption needs to be validated from the data analyzed $[4,5]$. For the ROIs inferred from rapid event-related fMRI data, the temporal features relevant to functional 
homogeneity should additionally include time evolution of the stimulus-response magnitudes over a fMRI session. With these considerations in mind, we extend several existing spatial-temporal fMRI models $[1,2]$ and propose a conceptually principled and computationally efficient methodology for inferring sub-ROI spatio-temporal patterns from rapid event related fMRI data.

A brief review of spatio-temporal modeling of fMRI data is given in the following. The major approaches to HRF modeling can be broadly grouped into parametric, semi-parametric, and non-parametric approaches. In a parametric approach [1], HRF is represented an analytical function with a small set of free parameters to be learned from the data. In a semi-parametric approach [3], the HRF is modelled using a small set of temporal basis functions. In a nonparametric approach [6], the entire HRF is to be estimated. As this estimation problem is obviously ill-posed, some smoothness constraints need to be imposed. A standard approach to spatio-temporal modelling of fMRI data spatially constrains (e.g. through Markov random field) mass univariate methods that model fMRI time series in individual voxels [1]. As alternative to spatially constraining individual voxel-based models, a spatial mixture of several localized 'prototypical' univariate temporal models has been considered in [2]. In comparison to the former approach, the latter one is computationally more efficient (less number of free parameters) and yields more interpretable models (each prototype can correspond to an underlying source of neural activation triggered by the stimulus). The proposed methodology assumes a number of characteristically different and spatially localised temporal patterns in fMRI data where each temporal pattern is characterised by an underlying HRF and a time series of stimulus-response magnitudes. By adopting a parametric approach to representing HRFs, a HRF is specified by three parameters, namely response onset, delay and duration. In our work, the estimation of these parameters is regularized by various biologically inspired constraints. This is of advantage, compared to the smoothness constraints imposed by $[4,5]$. In the proposed model, the response magnitude of each stimulus is treated as a free parameter. In [5], the response magnitudes are modeled as independent samples from an unknown Gaussian distribution. The distribution is learned from the data but the response magnitudes are marginalised out. In contrast, we can infer the hemodynamic response with a time-resolved manner. In $[4,5]$, the voxel space is subdivided into several clusters and all voxels of a cluster share either the same HRF or the same level of response magnitudes. In our model, however, several distinct temporal models are all responsible for explaining fMRI time series on a single voxel with the varying responsibility probabilities. At every time instance, one of these models is chosen in a probabilistic manner. This helps our method to better cope with non-stationarity in hemodynamic response, an important issue that was extensively addressed in [7]. The paper is organised as follows. After a brief introduction to spatio-temporal modelling of fMRI data (Sect. 1), we formulate our model and describe a numerical algorithm to learn model parameters in Sect. 2. In Sect. 3, the validation of our approach is presented using both synthetic and real data. The paper is concluded in Sect. 4. 


\section{Methodology}

Let a fMRI data set of $V$ voxels and $T$ volume (time steps) be denoted by a matrix $\mathbf{Y} \in \mathcal{R}^{V \times T}$, a fMRI time series at voxel $v$ by a vector $\mathbf{y}(v) \in \mathcal{R}^{T}$, a fMRI measurement at voxel $v$ and time $t$ by a scalar $y(v, t)$. Assume that $K$ temporal patterns could be observed in $\mathbf{Y}$. To formulate a spatio-temporal model for $\mathbf{Y}$, we first define the likelihood of $y(v, t)$ as follows

$$
p(y(v, t))=\sum_{k=0}^{K} p(k \mid v) \cdot p(y(v, t) \mid k)
$$

where index $k$ here represents a temporal model that could explain the $k$-th temporal pattern observed in $\mathbf{Y}$. The probability $p(k \mid v)$ is the prior probability for the $k$-th model being chosen to generate fMRI time series $\mathbf{y}(v)$ at voxel $v$ and $p(y(v, t) \mid k)$ is the probability for $y(v, t)$ being predicted by model $k$. Non-zero indices $k$ represent models that account for prototypical patterns originating from some spatially localised sources of neural activation; $k=0$ indexes a null model accounting for temporal patterns that are not related to any neural activation. Let $\Theta^{\text {STM }}$ denote a parameter set of the above model. Obviously, this set comprises of a set of spatial parameters and a set of temporal parameters, denoted by $\Theta^{\mathcal{S}}$ and $\Theta^{\mathcal{T}}$, which specify the probabilities $p(y(v, t) \mid k)$ and $p(k \mid v)$, respectively. The definition of $p\left(y(v, t) \mid k, \Theta^{\mathcal{T}}\right)$ and $p\left(k \mid v, \Theta^{\mathcal{S}}\right)$ are given below. As the temporal models are independent of voxel index $v$, they are considered as parametric model for $y(t)$. Further, it is assumed that except for the model with $k=0$, all temporal models share a canonical form. This canonical model is given as follows: 1) A fMRI time series $y(t)$ is composed of a signal component $x(t)$ and a noise component $\epsilon(t)$, i.e. $y(t)=x(t)+\epsilon(t)$; 2) The noise component $\epsilon(t)$ is modelled by white Gaussian noise with noise variance $\sigma^{2}$, i.e. $\epsilon(t) \sim \mathcal{N}\left(0, \sigma^{2}\right)$ We note that the assumption of i.i.d. noise can cause enhanced false-positive rate in activation detection $[2,8]$. However, as pointed out in $[2,8]$, clusteringbased methods (such as ours) are typically much less prone to false positives caused by the neglect of autocorrelation in fMRI noise; 3) The signal component $x(t)$ is given by $x(t)=\sum_{s=1}^{S} \sum_{\mathbf{p}=1}^{P} \mathbf{h}_{\mathbf{p}, s}(t)$, where $S$ is the total number of stimuli in a time window, $P$ is the number of cognitive processes evoked by a stimulus, and $\mathbf{h}_{\mathbf{p}, s}(t)$ represents the haemodynamic response of the $\mathbf{p}$-th process evoked by the $s$-th stimulus. For example, two separate cognitive processes (visual analysis process and perceptual judgement process) could be evoked by individual visual stimuli in a visual-perceptual experiment; 4) The haemodynamic response $\mathbf{h}_{\mathbf{p}, s}(t)$ is given by $\mathbf{h}_{\mathbf{p}, s}(t)=a_{\mathbf{p}, s} \cdot \delta\left(t-\left(t_{\mathbf{p}, s}+\tau_{\mathbf{p}, s}\right)\right) \otimes g_{\mathbf{p}, s}(t)$, where $a_{\mathbf{p}, s}$ is response magnitude, $t_{\mathbf{p}, s}$ is response onset, $\tau_{\mathbf{p}, s}$ is response delay, and $g_{\mathbf{p}, s}(t)$ represents response shape function. Moreover, $\delta()$ denotes delta function and $\otimes$ denotes convolution operator; 5) The response shape function $g_{\mathbf{p}, s}(t)$ is defined as a Gamma function $g(t)$ with its shape parameter $\kappa_{\mathbf{p}, s}$

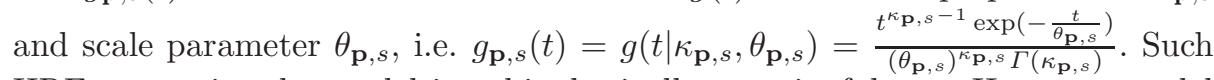
HRF constrains the model in a biophysically meaningful way. However, model 
estimation can be hampered by local maxima. We therefore combine careful parameter initialisation with biologically informed prior over HRF parameters (see below). We denote all haemodynamic response parameters by $\Theta_{\mathbf{h}}^{\mathcal{T}}$, that is, $\Theta_{\mathbf{h}}^{\mathcal{T}}=\left\{a_{\mathbf{p}, s}, \tau_{\mathbf{p}, s}, \theta_{\mathbf{p}, s}, \kappa_{\mathbf{p}, s},\right\}_{\mathbf{p}=1, \ldots, P} \quad{ }_{s=1, \ldots, S}$. Note that response onset $t_{\mathbf{p}, s}$ are known parameters and $\Theta_{\mathrm{h}}^{\mathcal{T}}$ is a $4 \cdot S \cdot P$-dimensional vector of free parameters. As we have $K$ temporal models of this canonical form, the $k$-th model is specified by its parameter set $\Theta_{\mathbf{h}, k}^{\mathcal{T}}$ and noise parameter $\sigma_{k}^{2}$. Its signal component is given by $x_{k}(t)=x\left(t ; \Theta_{\mathbf{h}, k}^{\mathcal{T}}\right)$, and the corresponding likelihood is $p\left(y(v, t) \mid k ; \Theta_{k}^{\mathcal{T}}\right)=\mathcal{N}\left(y(v, t) ; x_{k}(t), \sigma_{k}^{2}\right)$, with $\Theta_{k}^{\mathcal{T}}=\left(\Theta_{\mathbf{h}, k}^{\mathcal{T}}, \sigma_{k}^{2}\right)$ for $k \neq 0$. For the null model $(k=0)$, we have $x(t)=\mathbf{b}+\epsilon(t)$ with $\epsilon(t) \sim \mathcal{N}\left(0, \sigma_{0}^{2}\right)$, which accounts for a possible level shift of fMRI signal. Moreover, the shift is assumed to be constant over time (all low-frequency trends had been removed from the data). The corresponding likelihood is given by $p\left(y(v, t) \mid k=0 ; \Theta_{0}^{\mathcal{T}}\right)=$ $\mathcal{N}\left(y(v, t) ; \mathbf{b}, \sigma_{0}^{2}\right)$, with $\Theta_{0}^{\mathcal{T}}=\left(\mathbf{b}, \sigma_{0}^{2}\right)$. In summary, the set of temporal parameters $\Theta^{\mathcal{T}}=\left\{\Theta_{0}^{\mathcal{T}}, \Theta_{1}^{\mathcal{T}}, \ldots, \Theta_{K}^{\mathcal{T}}\right\}$. includes totally $K \cdot(4 \cdot S \cdot P+1)+2$ free parameters: $4 \cdot K \cdot P \cdot S$ haemodynamic response parameters, 1 level shift parameter, and $K+1$ noise parameters.

As pointed out in the previous subsection, the prior probability $p(k \mid v)$ varies across the region of interest. Clearly, it is an ill-posed problem to estimate $p(k \mid v)$ for every $v$. More importantly, it is known that evoked neural responses are spatially contiguous. Therefore, it is natural to impose smoothness constraints on the spatial variation of $p(k \mid v)$. Recall that $\Theta^{\mathcal{S}}$ denotes the set of spatial parameters that specify the spatial prior $p(k \mid v)$. Given voxel $v$, this prior probability is defined by the likelihood ratio $p\left(k \mid v ; \Theta^{\mathcal{S}}\right)=\frac{p\left(v \mid k ; \Theta_{k}^{\mathcal{S}}\right)}{\sum_{k=0}^{K} p\left(v \mid k ; \Theta_{k}^{\mathcal{S}}\right)}$, where $p\left(v \mid k ; \Theta_{k}^{\mathcal{S}}\right)$ is the probability that fMRI time series at voxel $v$, i.e. $\mathbf{y}(t)$ is generated by the $k$-th temporal model $\left(\mathbf{y}(t)=x_{k}(t)\right)$. Note that we have $\Theta^{\mathcal{S}}=\left\{\Theta_{0}^{\mathcal{S}}, \Theta_{1}^{\mathcal{S}}, \ldots, \Theta_{K}^{\mathcal{S}}\right\}$. This definition allows the smoothness constraints to be placed on $p(v \mid k)$ while ensuring that $\sum_{k=0}^{K} p(k \mid v)=1$.

Assume that the haemodynamic response of certain neural activation propagates from an epi-center across the whole ROI with certain covariance structure. Mathematically, this could be modelled by a three-dimensional Gaussian distribution. Hence, the likelihood is given by $p(v \mid k)=\mathcal{N}\left(\mathbf{r}_{v} \mid \mu_{k}, \Sigma_{k}\right)$, where $\mathbf{r}_{v}$ denotes the location of voxel $v, \mu_{k}$ is the mean vector of the Gaussian distribution, and $\Sigma_{k}$ is its covariance matrix. Note that we have $\Theta_{k}^{\mathcal{S}}=\left(\mu_{k}, \Sigma_{k}\right)$ for $k \neq 0$. For the null model $(k=0)$, we have $p(v \mid k=0)=\frac{1}{\mathcal{V}}$, where $\mathcal{V}$ is a free normalisation parameter, i.e. $\Theta_{0}^{\mathcal{S}}=\mathcal{V}$. This definition is rationalised by the assumption that the level shift of BOLD signals stays constant across individual ROIs. Note that $\mathcal{V}$ is not as same as the number of voxels, i.e. $V$ but ought to be larger than $V$. Otherwise, the null model could often dominate over the other models. This is because the spatial extent of ROIs is bounded and the probability mass of $p(v \mid k)$ over some ROIs could be significantly smaller than 1 . In summary, the set of spatial parameters $\Theta^{\mathcal{S}}$ includes totally $9 \cdot K+1$ free parameters: $3 \cdot K$ mean parameters, $6 \cdot K$ covariance parameters, and 1 normalisation parameter. 
In this work, a Bayesian approach is adopted to estimate all model parameters, i.e. $\Theta^{\text {STM }}$ that are used to specify our spatio-temporal model of fMRI data by maximizing the posterior distribution $p\left(\Theta^{\text {STM }} \mid \mathbf{Y}\right)=p\left(\mathbf{Y} \mid \Theta^{\text {STM }}\right) \cdot p\left(\Theta^{\text {sтM }}\right)$ where likelihood $p\left(\mathbf{Y} \mid \Theta^{\text {STM }}\right)$ and prior $p\left(\Theta^{\text {STM }}\right)$ are specified in what follows. Given our model, all fMRI measurements are conditionally independent in both spatial and temporal domains. Therefore, we have

$$
p\left(\mathbf{Y} \mid \Theta^{\mathrm{STM}}\right)=\prod_{v} \prod_{t} \frac{\sum_{k=0}^{K} p\left(v \mid k ; \Theta_{k}^{\mathcal{S}}\right) \cdot p\left(y(t, v) \mid k ; \Theta_{k}^{\mathcal{T}}\right)}{\sum_{k=0}^{K} p\left(v \mid k ; \Theta_{k}^{\mathcal{S}}\right)}
$$

Recall that $\Theta_{\mathbf{h}, k}^{\mathcal{T}}$ represents a set of haemodynamic response parameters that is used to specify the $k$ temporal model. Finally, the prior $p(\Theta)$ is factorized as follows: $p(\mathbf{b}) \cdot\left(\prod_{k=1}^{K} p\left(\Theta_{\mathbf{h}, k}^{\mathcal{T}}\right)\right) \cdot\left(\prod_{k=1}^{K} p\left(\sigma_{k}^{2}\right)\right) \cdot p(\mathcal{V}) \cdot\left(\prod_{k=1}^{K} p\left(\mu_{k}\right) p\left(\Sigma_{k}\right)\right)$, We further assume the same prior on $\Theta_{\mathbf{h}, k}^{\mathcal{T}}$ for all $k \neq 0$, i.e. $p\left(\Theta_{\mathbf{h}, k}^{\mathcal{T}}\right)=p\left(\Theta_{\mathbf{h}}^{\mathcal{T}}\right)$ which can be factorized as follows: $\prod_{s=1}^{S} \prod_{\mathbf{p}=1}^{P} p\left(a_{\mathbf{p}, s}\right) \cdot p\left(\tau_{\mathbf{p}, s}\right) \cdot p\left(\theta_{\mathbf{p}, s}, \kappa_{\mathbf{p}, s}\right)$. For most of the above parameters, no prior information is available because of large variability across a pool of fMRI data sets. Hence, their prior is set to an uniform distribution. For the variance parameter $\sigma_{k}^{2}$, its likelihood profile is normally flat for large $\sigma_{k}^{2}$. To make the estimation of this parameter robust, its prior is set to $p\left(\sigma^{2}\right) \propto \frac{1}{\left(\sigma^{2}\right)^{2}}$. Similarly, the prior of a covariance matrix $\left(\Sigma_{k}\right)$ is set to the so-called Jeffery prior, i.e. $p(\Sigma) \propto \frac{1}{|\Sigma|^{2}}$ where $|\Sigma|$ is the determinant of $\Sigma$. For the response delay parameter $\tau$, it is found in the previous EEG-informed fMRI study that $\tau$ varies roughly between 0.1 s and 0.3 s. Hence, a Gaussian distribution is used to represent this prior knowledge, with its mean equal to $0.2 \mathrm{~s}$ and its variance equal to 0.01 . For the response shape parameter $\kappa$ and $\theta$, we make use of its relation to so-called time-to-peak parameter $T$ and Full-width-at-half-maximum parameter $W$ of a Gamma function as follows $T=(\kappa-1) \theta$ and $W=\sqrt{2 \ln 2} \cdot \sqrt{\kappa} \theta$, respectively. It is reasonable to assume that the latency and duration of a haemodynamic response have an upper bound: $T_{\max }=4 \mathrm{~s}$ and $W_{\max }=8 \mathrm{~s}$. Thus, a logarithmic barrier function is used to represent this prior knowledge about the shape and scale parameter, that is, $p(\kappa, \theta) \propto \exp ^{-\log \left(T_{\max }-T\right)-\log \left(W_{\max }-W\right)}$. As seen in the previous two subsections, we have two subsets of model parameters to be learned from the data, those in temporal and spatial models. They are $\left\{\Theta_{k}^{\mathcal{T}}\right\}_{k=1}^{K}$ and $\left\{\Theta_{k}^{\mathcal{S}}\right\}_{k=1}^{K}$ respectively. In this work, we have developed a purpose-tailored algorithm to iteratively compute the Maximum A Posteriori (MAP) estimate of these 2 subsets of parameters. For each subset, a scaled conjugate-gradient optimization algorithm is employed. For any gradient-based optimization algorithms, only local optimum could be reached. The posterior distribution of a mixture-of-experts model could be highly multi-modal. Therefore, a good initialization is crucial. In this work, the clustering algorithm proposed in [8] is used to initialise the spatial prior of individual prototypes. Using this prior, we select a number of most representative voxels for each prototype. The temporal model of that prototype is initialised by fitting a canonical model to the data on those voxels. 

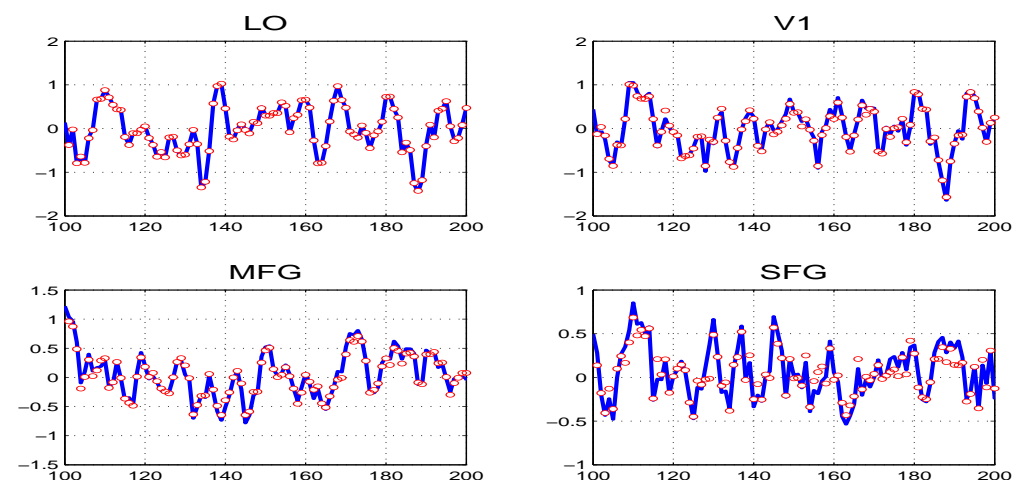

Fig. 1: Posterior estimates of BOLD signals (Red) and the fMRI measurements (Blue) as function of fMRI volume index for four different ROIs (LO, V1, MFG, and SFG).

\section{Results}

In this section, we first present some results in a controlled experimental setting using synthetic data that validate the algorithm developed for estimating parameters of our spatio-temporal model. Following this, we apply our algorithm to real fMRI data.

As pointed out previously, an artificial data set that resembles real fMRI data is generated to assess how accurate the model parameters can be learned from data when compared to ground truth values. The voxel space is $\{1,2, \ldots, 10\}^{3} \subset \Re^{3}$. We consider two prototypes (with the same spherical spatial prior $\Sigma=I$ ) of neural activation. In each of these 2 prototypes, two characteristically different but overlapping processes are set up. HRFs are the same, but the response amplitudes of prototypes 1 and 2 follow the sin and cos functions, respectively. Distance between the prototype means was varied from 6 to 1 . For estimating the spatial parameters, we use a non-informative initialisation - both prototype means are initialized at $(5,5,5)$ with the same spherical covariance matrix $3 I$. Further, we consider initialisation of temporal parameters as a deviation from the ground truth to some degree that varies from $1 \%$ to $30 \%$. For deviations up to $10 \%$ a good overall parameter estimation could still be achieved (generally, the spatial prior and temporal parameters were recovered to $1 \%$ and $3 \%$ precision, respectively), even with a non-informative initialisation of the spatial priors. Further, this performance can still be achieved when we initialise both spatial priors and temporal parameters with deviations up to $30 \%$.

Our real fMRI data are taken from a recent study in which participants were scanned while performing a categorization task on Glass pattern stimuli. The categorization task involved two processes: (1) visual perception and (2) motor response. Each scan run comprised 124 trials of 3s duration and EPI data were acquired with $\mathrm{TR}=1.5 \mathrm{~s}$. To illustrate our method, we select four subsets from 

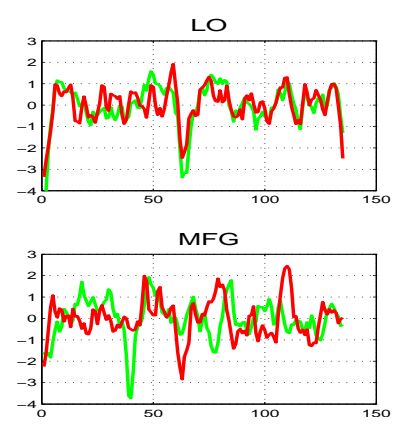
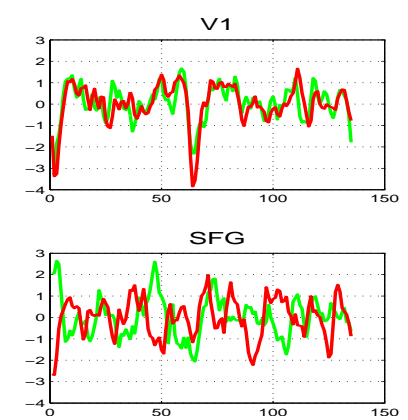

Fig. 2: Time evolution of response magnitudes of process 1 within the two prototypes in four different ROIs (LO, V1, MFG, and SFG).

the same data set of the same subject but corresponding to four different ROIs involved in visual perception: Middle Frontal Gyrus (MFG), Superior Frontal Gyrus (SFG), Primary Visual Cortex (V1), and Lateral Occipital Gyrus (LO). Note that MFG and SFG are two frontal ROIs whereas V1 and LO are two occipito-temporal ROIs. In the model to be learned from these data sets, the number of prototypes is fixed to two $(K=2)$. In each of these two prototypes, two processes are used to represent the processes evoked by the categorization task $(P=2)$. Once the MAP estimates of all model parameters are obtained, one can reconstruct the BOLD signal for each voxel in the corresponding ROI. Also, the HRFs can be reconstructed for each prototype-process pair in the model.

Fig. 1 shows that signal reconstruction is very satisfactory in all ROIs. Averaged over all voxels in individual ROIs, one can hardly detect any difference between the real measurements and the reconstructed signals. At the same time, it is found that the estimated HRFs are the same across different processes, prototypes and ROIs. We do not impose any constraints on non-stationary response magnitudes, making the model sufficiently flexible so that (for the given data) there is no need for different HRF shapes (even though the HRF free parameters would allow it). However, it is not necessary to see this lack of variability in HRFs across different ROIs as the shortcoming of our model because this kind of inter-ROI variability could also be characterised by comparing the time courses of response magnitudes between different ROIs. Fig. 2 shows that the time series of response magnitudes are temporally correlated. When AR model is used to explain these time series, the optimal AR order varies between 3 and 6 . Moreover, the response magnitudes of prototypes 1 are positively cross-correlated with those of prototype 2 in two occipito-temporal ROIs (V1 and LO). This observation could support the assumption that these ROIs are functionally homogeneous. For two frontal ROIs (MFG and SFG), however, the negative cross-correlation is observed. This can be seen as a strong indication of functional inhomogeneity in MFG and SFG. In summary, it is revealed that the sub-ROI spatio-temporal patterns are different between the occipito-temporal and frontal ROIs. 


\section{Conclusion}

We have presented a spatio-temporal model for ROI-based fMRI analysis. In this model, spatio-temporal behavior of fMRI time series is summarized by a small number of prototypical temporal patterns. Each temporal prototype comes with a spatial prior over the voxel space that determines its "region of influence" over voxels in its vicinity. We have validated our model with real fMRI data. The reconstructed BOLD signals agree with the real measurements on every voxel across the whole ROI. Due to the increased model complexity and difficulty in optimization, our method may not be able to cope with the cases with more than 5 prototypes, which is unlikely to occur in a ROI-based study.

Furthermore, we find that the occipito-temporal ROIs are functionally homogeneous and the frontal ones are not. This observation is confirmed by our follow-up study in which the fMRI data from totally 80 scan runs across 10 subject are analyzed. In that study, we also use the model selection method to determine the number of prototypes $(K)$ from the data. It turns out that two prototypes are needed to summarise the sub-ROI information in the frontal ROIs whereas one prototype is needed for the occipito-temporal ones. Actually, the determination of $K$ can be understood as a detection problem.

\section{Acknowledgements}

This work was supported by a grant to PT and ZK from the Biotechnology and Biological Sciences Research Council [H012508/1].

\section{References}

1. Svensen, M., Kruggel, F., von Cramon, D.Y.: Probabilistic Modeling of Single-Trial fMRI Data. IEEE Transaction on Medical Imaging 19 (2000) 25-35

2. Penny, W., Friston, K.: Mixtures of general linear models for functional neuroimaging. IEEE Transaction on Medical Imaging 22 (2003) 837-845

3. Woolrich, M.W., Behrens, T.E.J., Smith, S.M.: Constrained linear basis sets for HRF modelling using Variational Bayes. NeuroImage 21 (2004) 1478-1761

4. Hutchinson, R.A., Niculescu, R.S., Keller, T.A., Rustandi, I., Mitchell, T.M.: Modeling fMRI data generated by overlapping cognitive processes with unknown onsets using hidden process models. NeuroImage 46 (2009) 87-104

5. Chaari, L., Forbes, F., Vincent, T., Ciuciu, P.: Hemodynamic-informed parcellation of fMRI data in a joint detection estimation framework. Med Image Comput Comput Assist Interv. 15 (2012) 180-188

6. Marrelec, G., Benali, H., Ciuciu, P., Pelegrini-Issac, M., Poline, J.: Robust Bayesian Estimation of the Hemodynamic Response Function in Event-Related BOLD fMRI Using Basic Physiological Information. Human Brain Mapping 19 (2003) 1-17

7. Donnet, S., Laville, M., Poline, J.: Are fMRI event-related response constant in time? NeuroImage 8 (2006) 360-369

8. Heller, R., Stanley, D., Yekutieli, D., Rubin, N., Benjamini, Y.: Cluster-based analysis of FMRI data. NeuroImage 33 (2006) 599-608 